\title{
475374 - ANALYSIS BY HOLTER-ECG OF ARRYTHMOGENIC EFFECTS OF PROPOFOL VS SUPRANE FOR THE MAINTAINANCE OF GENERAL ANESTHESIA IN THE OBESE PATIENT
}

\author{
Ghania Khefif, Resident, Pierre Origer, MD, Constantinos Tsiberidis, resident \\ anesthesiology, Maurice Sosnowski, Professor \\ Anesthesiology, CHU St Pierre, Brussels, Belgium
}

Introduction: Perioperatively 60 to $90 \%$ of surgical patients present cardiac arrythmias. Wang and all(1) provided evidence that obesity is an important risk factor for arrythmias in the adult. Suprane is known to trigger the autonomic nervous system and could possibly enhance arrythmias in risk groups. The aim of this study was to evaluate the incidence of arrythmias, comparing propofol and suprane maintainance in obese patients. Methods: Our study was approved by the Research Ethics Board of our hospital and we obtained informed consent from 46 patients scheduled for bariatric surgery. Patients with a body mass index $>35 \mathrm{~kg} / \mathrm{m} 2$ were randomly assigned to maintainance by propofol (Group A; n=21) or suprane (Group B; n=19). We monitored all patients by Holter-ECG 24 hours prior to surgery (Period 1=Pd1) and 24 hours following surgery(Period 2=Pd2)

Results: Mean heart rate (MHR) rose significantly after surgery for all patients $(\mathrm{A}+\mathrm{B})$ with $p=0.0018$. Within Group A, MHR was significantly higher during Pd 2 ( $p=0.0107)$. There was a near significant rise in MHR for group B during Pd2. Mean heart rate variability (MHRV) within Group A did not change significantly between Pd1 and 2 $(p=0,20)$. However group B presented a statistically significant narrowing of MHRV $(p=0.0266)$. We noted no differences in the occurance of supra ventricular and ventricular arrythmias.

Discussion: Suprane is responsible for narrowing of MHRV. Depressed heart rate variability is an independant predictor of mortality and arrythmic complication in cardiac pathologies(2). We suspect that suprane could possibly trigger postoperative arrythmias in the obese patient. We did not observe more arrythmic incidents with suprane in our study, the reason might be the small number of cases.

References: 1 .Wang TJ and all. JAMA 2004;292:2471-77 2. Task force of the ESC the NAS of Pacing Electrophysiology.Circulation.1996;93:1043-65

Results 


\begin{tabular}{|c|c|c|c|}
\hline MHR & & & \\
\hline Group A & Pd 1 & $77,28 \pm 1,90$ & \multirow{2}{*}{$\mathrm{p}=0.0107^{*}$} \\
\hline & Pd 2 & $84,67 \pm 2,50$ & \multirow{2}{*}{$\mathrm{p}=0.053$} \\
\hline Group B & Pd 1 & $78,82 \pm 3,53$ & \\
\hline MHRV & Pd 2 & $78,82 \pm 3,53$ & \multirow{2}{*}{$\mathrm{p}=0.20$} \\
\hline Group A & Pd 1 & $77,5 \pm 5,39$ & \\
\hline & Pd 2 & $69,11 \pm 5,48$ & \\
\hline Group B & Pd 1 & $92,11 \pm 5,26$ & \\
\hline & Pd 2 & $72,18 \pm 5,13$ & \\
\hline
\end{tabular}

